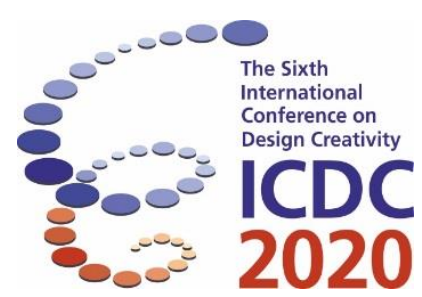

\title{
Analysing Divergent-Convergent Activities in the Architectural Studio, with the aid of the 'Knowledge Construction Activities' model
}

\author{
Hadas Sopher
}

Faculty of Architecture and Town Planning, Technion - Israel Institute of Technology, Haifa, Israel

\begin{abstract}
Design activities and divergent-convergent developmental activities are considered to be of essential importance in developing design solutions, making them an important learning objective in the architectural studio. However, since most studio assessments focus on the quality of the artefact, learners are not provided with adequate feedback as to their achievements in applying these activities during the learning process. In response, this study utilizes the "Knowledge Construction Activities" (KCAs) model as a means to measure the application of the stated activities in learners' performance throughout the course. Learners' design decisions and the modifications applied upon them to generate design development are coded into measurable learning graphs, making it possible to identify peaks of divergent-convergent activity as well as reduced practice. A case study of three undergraduates highlights in detail each subject's performance, providing the means to support a custom-tailored approach to teaching.
\end{abstract}

Keywords: KCA analysis, studio, divergent convergent activities, creativity, learning analytics

\section{Introduction}

One of the primary goals of the architectural studio is to support students in gaining proficiency in design activities required in the development of a design solution. To do so, tutors need a detailed picture of students' achievements in practicing design activities throughout the course. However, the lack of means to depict performance creates a gap in the tutor's information, leading to a shortcoming in providing learners with adequate feedback as to the activities successfully practiced as well as the ones which require further support. To address this need, this study depicts the way individual learners perform at a longitudinal learning process, done at the studio. The author utilizes the Knowledge Construction Activity (KCA) model of analysis, developed in previous studies (Sopher, Kalay, \& Fisher-Gewirtzman, 2017; Sopher, Fisher-Gewirtzman, \& Kalay, 2019), in order to determine the different types of activities practiced throughout a course by individual learners.

\subsection{Design Activity}

Cross (2006) describes designers' constructive knowledge, referring to a set of activities, required to form new ideas and develop existing decisions in order to achieve a solution. Design activities are known to include of analysis, synthesis and evaluation type of activities, which involve the structuring of the problem, generating solutions in and testing them (Goel \& Pirolli, 1992). It follows that each decision is generated by the application of a specified type of activity. The development of existing 
decisions occurs through what are referred to as divergent-convergent activities, two mechanisms of thought that are associated with producing design alternatives and creating solutions. Gabora (2010) defines divergent activities as associative thoughts able to expand one's attention, while convergent activities focus that attention. Shifts between these modes of thought determine how a problem is structured. Gabora (2018) describes how the individual's worldview affects the emergence of divergent and convergent activities. Accordingly, divergent thought seeks to expand the context, or the solution considered, by creating new alternatives. This mode of thought makes it necessary to step back from the known context, a step that requires extensive activity, such as forming new connections between previously unrelated issues. Convergent activity is described not only as consolidating two or more decisions, but also as making it necessary to choose the set of pertinent alternatives within the context. Described by Goel (2014), divergent activities broaden the scope of the problem through the creation of associative decisions, making it possible to move beyond preconceptions and the mere structuring of the problem, while inferences developed from existing decisions support convergent activity, resulting in greater detail or a new relationship. Based on these explanations, divergence is seen as an activity that expands a design decision in more than one trajectory, while convergent activity involves deep inference of multiple issues until arriving at a single solution. It follows that decisions containing different types of design activity can be developed by applying divergence or convergence activities. The expansion of the problem with additional subproblems or goals can be considered as the divergence of the analysis activity, while the development of associative solutions describes the divergence of the synthesis activity. Divergent and convergent activities play critical roles in achieving progress in design, by producing multiple alternatives for the convergence of different concepts (Dorst \& Cross, 2001), while also fostering the formulation of successful ideas (Georgiev \& Georgiev, 2018). Tversky and Chou (2011) hold that applying numerous divergent activities contributes to avoiding fixation during the design process.

Divergent-convergent activities are acknowledged to associate with creative thinking (Mednick, 1962), a desired way of thought in the field of design for the need to produce new solutions. Tracking these activities during design activity serves as evidence of creative thinking (Goldschmidt, 2016). Similarly, the extensive production of specifications (Kim, Jin, \& Lee, 2011) or issues discovered during interaction with design artefacts (Gero \& Kan, 2016) indicate on creative thinking, all of which can be considered as the divergence of decisions. Rosenman and Gero (1993) propose procedures of design development as the criteria for identifying creativity by considering the changes expressed in design artefacts as evidence of how decisions have either combined with one another or mutated, evidence which can be considered as the convergence of decisions. These studies point to an evolving approach which holds that gaining more control over design performance can enhance creative thinking and support design progress, significant factors when it comes to educating students.

\subsection{Learning how to design}

The studio course serves as the core setting for learning how to design. Under the experiential learning approach (Dewey, 1938/2007), students construct design knowledge by independently interacting with an architectural problem, which they ultimately develop into a final design artefact through practice of design activities (Schön, 1987). Such a task makes no easy challenge. While it is extremely important for students to gain hands-on experience with design activities, they may find it difficult to apply design activities and develop their decisions. Design problems are viewed as 'wicked' problems (Rittel \& Webber, 1973). They have no determined solution, making progress to be highly dependent on personal judgment. In addition, each decision has an impact on subsequent decisions. Such criteria may pose difficulties in applying divergent or convergent activities to existing decisions. Some students may produce design alternatives, but find it challenging to make them converge. In other cases, students may struggle to expand a concept to facilitate progress. Compared to professional designers, novices tend to produce more design issues, but organise them less effectively or in a lesser depth (Becker et al., 2018), showing the difficulty in treating decisions with divergent-convergent activities. Since divergentconvergent thinking modes require extensive decision making (Gabora, 2018), it is important to support students in practicing these activities so as to gain proficiency. 
To ensure practice, most studio courses foster a longitudinal learning process that lasts a semester or two, conducted in several social modes of assessment: A personal, tutor-learner crit; Group crits expand the sources of knowledge through peer participation; Mid-term and final reviews expand the group setting to include professional guests, providing students with a summative assessment as to their achievements in developing a solution. By making a significant contribution to the generation of new ideas (Goldschmidt et al., 2010), studio crits play a key role in advancing learning activities. Scalone, Atman, Twigg-smith, Shroyer, and Joya (2020) hold that questions that arise during the design process support designers in better engaging with divergent and convergent activities. In this sense, crit discussions, group crits and reviews in particular, become a significant stimuli. Due to the educational role of reviews, heightened by the fact that guests who are not familiar with the learner's work are in attendance, such crits enhance learning activities, stimulating learners to better explain (and express) their ideas. It is to be expected, therefore, that reviews will be characterised by higher levels of convergent activity while triggering divergent activity (in the case of mid-term reviews).

\section{The studio's shortcoming in measuring learners' performance}

The aforementioned studies show how important it is for students to gain proficiency in applying design activities. However, given that the most common type of studio assessments focuses on the quality of the artefact (De la Harpe et al., 2009), students are not receiving sufficient feedback on their achievements (or failures) in the practice of design activities. Due to their wicked characteristics (Rittel $\&$ Webber, 1973), design problems are unique, meaning that the number of possible solutions for a given design problem is infinite. It follows that the quality of the solution may not be a good indicator of the extent to which a student practiced design activities. Furthermore, though a successful artefact may imply a general level of competence, it does not show which activities still require further practice, thus limiting the tutors' ability to provide adequate feedback and to promote learners in accordance with their abilities.

The extant focus on crit communication events held in research provides limited information on students' performance. Analyses of cognitive processes during crit conversations highlighted the role of crits in placing the focus on design solutions, as well as the extent to which tutors dominate crit conversations (Milovanovic \& Gero, 2018). Pauwels, Strobbe, Derboven, \& De Meyer (2015) observed that many repetitions occurred during the conversation, a pattern found to be denser than in cycles that occurred during non-educational design activities. These studies show that crit conversations may not depict the cycle of activities practiced by students, leading to the need for a method able to depict learner performance, as a variable independent of crit conversations or the quality of the artefact.

Noteworthy efforts have been made to study the cognitive processes and steps that take place during design activity, making it possible to analyse one's proficiency in applying certain activities. Extant methods have successfully identified evidence of divergent-convergent activities (Georgiev \& Georgiev, 2018), critical decision-making and iterative cycles of divergent-convergent activities (Goldschmidt, 2016) and the distribution of design issues and their development (Becker et al., 2018). Meant to analyse the design process in terms of its micro-steps of change, these methods depict a design event through a continuous conversation cluster, or a part of one. They do not show how a certain decision may develop in different time-frames, as often occurs in design-learning processes. The crits of a studio course are linked together in a continuous learning process. Consequently, a decision may diverge into several decisions in different following crits. Assessing design activity across crits is of critical importance when the aim is to provide students with proper feedback as to their achievements throughout the course.

\section{The Knowledge Construction Activity model}

With the aim to assess learners' abilities in applying design activities, this study utilizes the KCA model (Sopher et al., 2017; Sopher et al., 2019) as means to study individual performance. The model allows to represent and quantify the design activities practiced by students across crits. The design decisions prepared by the learner and presented in a given crit are considered as KCA units, consisting of information about the type of activity practiced and the type of social construct in which it was 
discussed. Links between units point to a modification in a given decision, thereby serve evidence that design development activity took place.

A KCA describes a decision containing a new issue or a change that was applied to, or that resulted from previous decisions, serving as evidence of learning activity. Each KCA consists of a decision domain and definition that articulate an issue addressed in dealing with the design problem (E.g., a façade). The type of activity is determined in accordance with the principles elaborated by Goel \& Pirolli (1992). Analysis consists of recognizing problems or formalising goals and constraints. Synthesis relates to changes applied to the artefact in terms of its form and function. Evaluation refers to tests made to study the artefact's ability to meet the goals set, such as energy or structural tests, comparisons of design alternatives, and assessments of the extent to which limitations, such as height or density, are compatible with the desired solution. The type of activity is identified in the outcomes prepared by the learner. (Figure 1). In order to depict activity in different crit models (E.g., a personal crit), each KCA contains information on the social construct in which a decision was discussed. Table 1 outlines the KCA coding scheme.

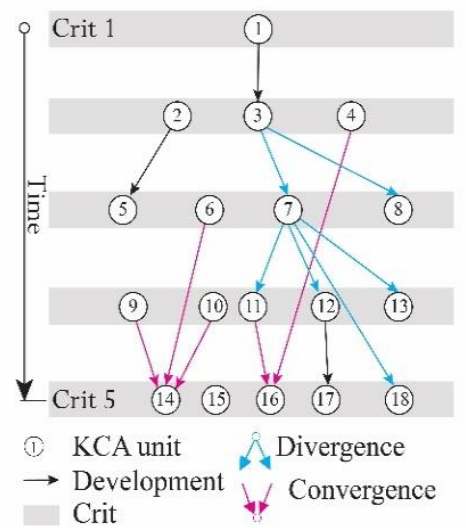

Figure 1: a KCA learning graph

Table 1: KCA coding scheme

\begin{tabular}{|c|c|c|}
\hline KCA component & & Description \\
\hline KCA label & & Chronological number of each decision. E.g., KCA-1 \\
\hline Crit & & Chronological number of each crit, to represent time \\
\hline Decision & Domain & A design problem. E.g. west façade \\
\hline & Definition & Description of the design act. E.g., setting the size of the windows \\
\hline Design activities & & Analysis, Synthesis, Evaluation \\
\hline Social setting & & Crit assessment model. E.g., a group crit \\
\hline
\end{tabular}

A linking method is utilized to illustrate the way KCAs are developed. Developmental activities are represented as vectors that link two or more KCAs and thus contain both a source and a target (Figure 2). A vector's connection to a subsequent KCA is determined by the modifications applied to previous KCAs. A development is identified in accordance with the principles formulated by Goel (2014), and Alexander (1964), with the rules being applied to an existing decision while creating possible new solutions, or formulating new subproblems. Alexander (1964, pg. 62) describes design development in terms of the increasing number of requirements that need to be resolved. Under these principles, development links point to further progress made in the domain of a decision (e.g., a building may have details added to its façade to meet further requirements) or to application of a rule being extended to another subject (e.g., applying a grid module of one form to other forms). The learning timeline is represented by the vertical direction of crit clusters (Figure 1). All the KCAs presented during a given crit are represented in a single crit cluster. Since development is expected to occur as the course progresses, links between decisions in different clusters are a significant indicator that this has indeed taken place. 
It should be noted that the KCA approach analyses learners' visual outcomes as expressions of design knowledge (Bresciani, 2019). As such, these outcomes reflect the activities a learner is able to apply, while also indicating the ones that are poorly applied. In contrast, protocol analysis techniques (Krauss, Hall, Fussell, \& Hall, 1996), though successfully used to analyse design activity (Cross, 2001), fail to indicate how learning progresses across crits, nor can they point to peaks (or instances of reduced activity) along the way. Demonstrated in figure 2, are the types of design activity, identified in the outcomes: (a.) A circulation scheme is identified as an analysis activity. (b.) A synthesis activity is identified by the two volumes developed from two surfaces. The development is coded as a link between the two decisions (c.) The light assessment is identified as an evaluation activity.
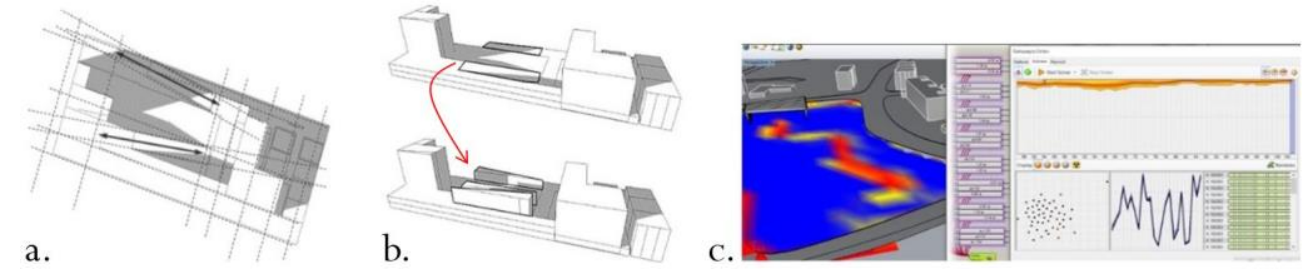

Figure 2: Identifying types of design activities in the learners' outcomes

\subsection{Identifying Divergent and Convergent Activities}

Once KCAs and links are coded, it is possible to track divergent and convergent activities in the graph generated. Illustrated in figure 1, divergent activities are seen in a KCA containing two source nodes (or more), indicating that a single decision has expanded into several decisions. A convergent activity is tracked when a KCA receives the targets of two vectors (or more), indicating that several decisions have converged into a single one. Analysis of divergent activities will count the number of source nodes, whereas the number of converged end nodes will elucidate the number of convergence activities practiced during the course or in a single crit. Previous studies made by this author, utilized the KCA model to investigate how different learning environments impact the way learners apply design activities (Sopher et al., 2017) and divergent-convergent activities (Sopher et al., 2019), revealing that learners used different environments to support different types of activity. This study expands previous understandings by exploring the model's ability to depict the types of design activity being diverged or converged.

\section{Case Study}

Meant to describe individual learning performance, this study follows three undergraduate students (ages 20-25) in their third year of study in the same studio course at the Faculty of Architecture and Town Planning, Technion, Israel. The subjects were monitored during an entire course that included twenty crits, through non-interfering observations and crit recording. The course consisted of three reviews, occasions on which professional guests were present. The assignment called for the design of the re-utilisation of an existing public building, including a new program of functions and design solutions. The design decisions presented at each crit were documented in order to identify the KCAs and trace the developmental links. KCA coding is done with Gephi, visual analytics software that is publicly available (Bastian, Heymann, \& Jacomy, 2009), making it possible to analyse the data in accordance with the above-stated components. All data collection and analyses were conducted by this author.

\section{KCA Analysis}

The analysis of three students retrieved $366 \mathrm{KCAs}$, generated in three individual learning graphs. Table 2 depicts each learner's production of KCAs, showing that most design activity is of the synthesis type. Differences in design activity were found between the subjects. Student S01 extensively applied synthesis activities, meaning that the learning performance was highly solution-focused. The reduced number of analysis activities indicates a need for additional practice. The students S02 and S03 achieved a similar percentage of activities. Repeating the study upon more subjects will potentially contribute to 
the understanding of commonalities in the practice of design activities during the learning process. In all three cases, evaluation activities were rarely practiced.

Table 2: Learners' production of KCAs in accordance with activity types

\begin{tabular}{ccccc}
\hline students & KCAs & Analysis (\%) & Synthesis (\%) & Evaluation (\%) \\
\hline S01 & 103 & 25.24 & 71.84 & 2.91 \\
S02 & 140 & 41.43 & 55.71 & 2.86 \\
S03 & 117 & 38.46 & 59.83 & 1.71 \\
\hline
\end{tabular}

Figure 3 depicts the number of divergent and convergent activities applied to KCAs in accordance with their type of design activity. All Students show a prevalence of divergent activities, while a lesser amount of convergent activities was applied throughout the course. Similar ratios were found in continuous design clusters (Goldschmidt, 2016). Findings reveal that most KCAs were not diverged nor converged, pointing to the difficulties faced by learners in practicing these activities. Student S01 diverged twenty-six KCAs, which form $25 \%$ of her total production of decisions while converging $13.6 \%$ of KCAs. Student S02 diverged 19\% of KCAs and converged $11.4 \%$, and student S03 diverged $23 \%$ and converged $15.3 \%$ of KCAs. Both divergent and convergent activities were applied primarily to synthesis KCAs, revealing the focus of all learners on developing existing solutions. Compared to the two other students, Student S01 experienced more difficulties in investigating existing problems and setting goals. The student changed the program of functions several times and had troubles in creating connections between issues. Consequently, she achieved a reduced amount of the analysis type of KCAs that were either diverged or converged, indicating a need for adequate tutor guidance.
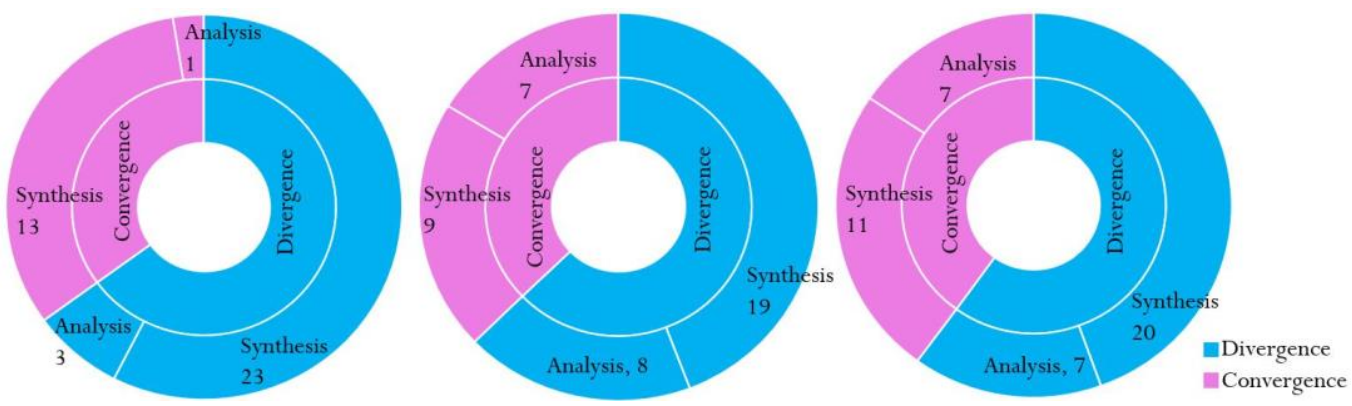

Figure 3: Divergence or convergence activities applied to KCAs in accordance with the type of design activity. Student S01 (left), S02 (middle), S03 (right)

Figure 4 presents each subject's production of divergence and convergence activities throughout the course. All three analyses show iterative cycles of divergent-convergent activities, indicating the constant practice of these activities. These results are consistent with the findings of Goldschmidt (2016), while also illustrating the way the cycles evolved throughout the course. Student S01 achieved a small number of divergent-convergent activities at the beginning of the semester, which was followed by two events of intensive design development, divergence in particular, one at mid-semester (crits 912) and another near the final review (crits 18-23). The design decisions of student S02 were mainly developed between the second and the final reviews (crits 15-23), presenting iterative cycles of divergent-convergent activity. Such results may point to improved performance as the semester progressed. Student S03 had a single episode of intense design development, between the crit number nine and the second review (crit 15), followed by a significant decrease in iterative cycles, reflecting a need for attentive tutor support. The student experienced fixation on a well-converged concept, achieved during the second review, while having trouble in further diverging it. Following the conclusions stated by Tversky and Chou (2011), encouraging the student to apply divergent-convergent activities may have helped her to avoid fixation. 


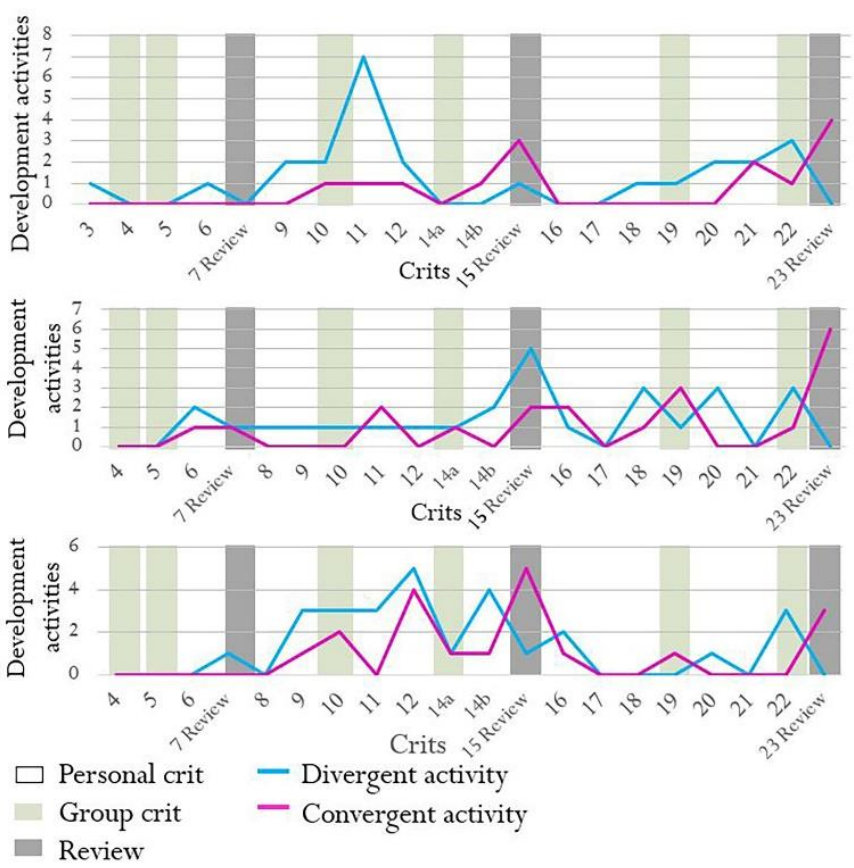

Figure 4: Performance of divergence-convergence activities throughout the course. Student S01 (top), S02 (middle), S03 (bottom).

Examining performance in terms of the different social constructs of the crits revealed differences in the performance of divergent and convergent activities (Figure 4). As expected, the reviews, and especially the second and final ones, were characterised by increased convergent activity. Surprisingly, the mid-term review did not result in a significant increase in divergent activity during the following crit, nor did it lead the students to apply convergent activities. These results can be explained by the noteworthy decrease in the production of KCAs on the occasion of the following crit, found in all the subjects. Although a decrease in productivity is common following mid-term reviews, it raises the question of the effectiveness of this social setting to support students in developing their artefacts and calls for further investigation of this issue. Student S03 primarily applied divergent-convergent activities after personal crits, while students S01 and S02 had an increased number of divergent activities after group crits, indicating how they benefited the setting for expanding existing concepts, suggesting that diversity in the educational social setting may support different learners.

\section{Discussion}

This study describes how students practice design activities and divergent-convergent developmental activities during the architectural studio, with the aid of the KCA model to analyse individual performance. The results of three case studies provide a detailed picture of the activities practiced throughout the entire duration of the course. The findings point to an apparent prevalence of decisions of synthesis type being applied, further developed by divergent activities. A lesser number of convergent activities was observed, indicating that gaining such a proficiency may require greater tutor support.

The findings point to evidence of iterative cycles of divergent-convergent activity throughout the semester, deemed to be essential for the development of artefacts and creativity (Dorst \& Cross, 2001). This behaviour pattern is consistent with the previous analysis of divergent-convergent activity made by expert designers (Goldschmidt, 2016), while providing analyses of an entire course, as well as to the type of activities that were either diverged or converged. The reduced number of KCAs of the type analysis which was developed indicates the need for adequate tutor support. Previous studies found crit conversations to be solution-focused (Milovanovic \& Gero, 2018). It follows that studio pedagogy ought to pay attention to the practice of additional activities rather than promoting a design solution. 
Examining learners' performance based on different types of crits, pointed to increased convergent activity in the proximity of reviews, followed by a decrease in activity, indicating that these crits did not have a significant impact on the subsequent design development. Since personal crits are known to be tutor-centric (Goldschmidt et al., 2010), group crits, found in this study to encourage divergent activity, may become a significant setting for expanding sources of knowledge. Although the sample examined is small, this study demonstrates how the KCA model can become a useful tool to depict the implications of the educational setting on learner performance.

The KCA model provides the means to produce evidence of the activities practiced during a given course, in addition to identifying those that were not practiced enough; this is information that can contribute to identifying the learner's strengths and weaknesses, while support a custom-tailored approach to teaching. It is to be hoped that, with the aid of the KCA model, studio instruction can address these critical issues, in order to provide adequate support for learners' needs.

\section{Acknowledgements}

Yehuda E. Kalay is greatly acknowledged for providing a broad perspective and key insights. This study could not be accomplished without the support of Dafna Fisher-Gewirtzman, who generously opened the gates of her course and the students who shared their design projects. The research was supported by the European Research Council grant (FP-7 ADG 340753) and by the Jacobs scholarship.

\section{References}

Alexander, C. (1964). Notes on the synthesis of form. Harvard University Press.

Bastian, M., Heymann, S., \& Jacomy, M. (2009). Gephi: An Open Source Software for Exploring and Manipulating Networks. In The Third International AAAI conference on weblogs and social media. (pp. 361362). San Jose. https://doi.org/10.1136/qshc.2004.010033

Becker, K. H., Gero, J. S., Pourmohamadi, M., Abdellahi, S., De Souza Almeida, L. M., \& Luo, Y. (2018). Quantifying differences between professional expert engineers and engineering students designing: Empirical foundations for improved engineering education. ASEE Annual Conference and Exposition, Conference Proceedings, 2018-June.

Bresciani, S. (2019). Visual Design Thinking: A Collaborative Dimensions framework to profile visualisations. Design Studies, 63, 92-124. https://doi.org/10.1016/j.destud.2019.04.001

Cross, N. (2001). Design cognition: results from protocol and other empirical studies of design activity Book Section Chapter 5 Design Cognition: Results From Protocol And Other Empirical Studies Of Design Activity. In C. Eastman, W. Newstatter, \& M. McCracken (Eds.), Design knowing and learning: cognition in design education (pp. 79-103). Oxford.

Cross, N. (2006). Designerly ways of knowing. London: Springer.

De la Harpe, B., Peterson, J. F., Frankham, N., Zehner, R., Neale, D., Musgrave, E., \& McDermott, R. (2009). Assessment Focus in Studio: What is Most Prominent in Architecture, Art and Design? International Journal of Art \& Design Education, 28(1), 37-51. https://doi.org/10.1111/j.1476-8070.2009.01591.x

Dewey, J. (1938). Experience and education. New York: Simon and Schuster (Original work published 1938).

Dorst, K., \& Cross, N. (2001). Creativity in the design process: co-evolution of problem-solution. Design Studies, 22(5), 425-437. https://doi.org/10.1016/S0142-694X(01)00009-6

Gabora, L. (2010). Revenge of the "Neurds": Characterizing creative thought in terms of the structure and dynamics of memory. Creativity Research Journal, 22(1), 1-13. https://doi.org/10.1080/10400410903579494

Gabora, L. (2018). Reframing Convergent and Divergent Thought for the 21st Century. (No. arXiv preprint arXiv:1811.04512).

Georgiev, G. V., \& Georgiev, D. D. (2018). Enhancing user creativity: Semantic measures for idea generation. Knowledge-Based Systems, 151, 1-15. https://doi.org/10.1016/j.knosys.2018.03.016

Gero, J. S., \& Kan, J. T. (2016). Empirical Results From Measuring Design Creativity: Use Of An Augmented Coding Scheme In Protocol Analysis. In The Fourth International Conference on Design Creativity (4th ICDC). Atlanta.

Goel, V. (2014). Creative brains: designing in the real world. Frontiers in Human Neuroscience, 8(241), 1-14. https://doi.org/10.3389/fnhum.2014.00241

Goel, V., \& Pirolli, P. (1992). The structure of design problem spaces. Cognitive Science, 16(3), 395-429. https://doi.org/10.1016/0364-0213(92)90038-V 
Goldschmidt, G. (2016). Linkographic Evidence for Concurrent Divergent and Convergent Thinking in Creative Design. Creativity Research Journal, 28(2), 115-122. https://doi.org/10.1080/10400419.2016.1162497

Goldschmidt, G., Hochman, H., \& Dafni, I. (2010). The design studio "crit": Teacher-student communication. Artificial Intelligence for Engineering Design, Analysis and Manufacturing, 24(03), $285-302$. https://doi.org/10.1017/S089006041000020X

Kim, Y. S., Jin, S. T., \& Lee, S. W. (2011). Relations between design activities and personal creativity modes. Journal of Engineering Design, 22(4), 235-257. https://doi.org/10.1080/09544820903272867

Krauss, R. M., Hall, S., Fussell, S. R., \& Hall, M. (1996). Social psychology models of interpersonal communication. In E. Higgins \& A. Kruglanski (Eds.), Social psychology: Handbook of basic principles (pp. 655701). New York: Guilford Press.

Mednick, S. A. (1962). The Associative Basis of the Creative Process. Psychological Review, 69(3), $220-232$.

Milovanovic, J., \& Gero, J. S. (2018). Exploration of cognitive design behaviour during design critiques. In Proceedings of International Design Conference, DESIGN (Vol. 5, pp. 2099-2110). https://doi.org/10.21278/idc.2018.0547

Pauwels, P., Strobbe, T., Derboven, J., \& De Meyer, R. (2015). Conversation and Critique within the Architectural Design Process: A Linkograph Analysis. In J. Gero (Ed.), Design Computing and Cognition (pp. 135-152). Springer, Cham.

Rittel, H. W. J., \& Webber, M. M. (1973). Dilemmas in a general theory of planning. Policy Sciences, 4(2), 155169. https://doi.org/https://doi.org/10.1007/BF01405730

Rosenman, M. A., \& Gero, J. (1993). Creativity in Design Using a Design Prototype Approach. In J. S. Gero \& M. Lou Maher (Eds.), Modeling Creativity and Knowledge-based Creative Design (pp. 111-138). New Jersey: Lawrence Erlbaum Associates.

Scalone, G., Atman, C. J., Twigg-smith, H., Shroyer, K., \& Joya, A. (2020). Dealing with Ambiguity : Leveraging Different Types of Expertise to Guide Design Questioning *. International Journal OfEngineering Education, 36(2), 773-795.

Schön, D. A. (1987). Educating the Reflective Practitioner. Toward a new design for teaching and learning in the professions. (first). San Francisco: Jossey-Bass.

Sopher, H., Fisher-Gewirtzman, D., \& Kalay, Y. E. (2019). Going immersive in a community of learners? Assessment of design processes in a multi-setting architecture studio. British Journal of Educational Technology, 50(5), 2109-2128. https://doi.org/10.1111/bjet.12857

Sopher, H., Kalay, Y. E., \& Fisher-Gewirtzman, D. (2017). Why Immersive? - Using an Immersive Virtual Environment in Architectural Education. In A. Fioravanti, S. Cursi, S. Elahmar, S. Gargaro, G. Loffreda, G. Novembri, \& A. Trento (Eds.), The 35th eCAADe Conference (pp. 313-322). Rome: CumInCAD.

Tversky, B., \& Chou, J. Y. (2011). Creativity: Depth and Breadth. In Design Creativity 2010 (pp. 209-214). London: Springer London. https://doi.org/10.1007/978-0-85729-224-7_27 